\title{
Design and Simulation of Hartley based Multi Orthogonal Band OFDM
}

\author{
Ali T. Shaheen \\ University of Baghdad/College of Eng. /Electrical Eng. Dept.
}

\begin{abstract}
Recent advances in wireless communication systems have made use of OFDM technique to achieve high data rate transmission. The sensitivity to frequency offset between the carrier frequencies of the transmitter and the receiver is one of the major problems in OFDM systems. This frequency offset introduces inter-carrier interference in the OFDM symbol and then the BER performance reduced. In this paper a MultiOrthogonal-Band MOB-OFDM system based on the Discrete Hartley Transform (DHT) is proposed to improve the BER performance. The OFDM spectrum is divided into equal subbands and the data is divided between these bands to form a local OFDM symbol in each sub-band using DHT. The global OFDM symbol is formed from all sub-bands together using the (IDHT). The BER performance of the proposed system is simulated and compared with the conventional OFDM in different channel conditions to show the gain in SNR achieved by the proposed system.
\end{abstract}

\section{Keywords}

Discrete Hartley Transform (DHT), OFDM and Multi Orthogonal Band OFDM

\section{INTRODUCTION}

The bandwidth and quality of service are very important parameters in modern communication systems, and service providers are frequently met with the challenge of increasing the users and services with in a limited allocated bandwidth. The OFDM (orthogonal frequency division multiplexing) is used to increase data rate of wireless medium with higher performance, [1]. OFDM is special form of multi-carrier transmission technique where a single high rate data stream is divided into multiple low rate data streams. These data streams are then modulated using subcarriers which are orthogonal to each other. The main concept of the OFDM is based on the Orthogonality between the subcarriers. The property of orthogonality allows simultaneous transmission of a lot of sub-carriers in a narrow frequency space without interference from each other. By this way the symbol rate on each sub channel is greatly reduced, and then the inter symbol interference (ISI) due to channel dispersion in time caused by multipath delay spread is reduced. For more ISI reduction, Guard interval can also be inserted between OFDM symbols $[2,3]$.

The orthogonalitybetween the subcarriers in an OFDM symbol is maintained only if the channel is flat and timeinvariant, channels with a Doppler spread and the corresponding time variations corrupt the orthogonality of the OFDM sub-carrier waveforms [4]. In a dispersive channel, self-interference occurs among successive symbols at the same sub-carrier causing Inter Symbol Interference (ISI), as well as among signals at different sub-carriers casing Inter Carrier Interference (ICI). For a time invariant but frequencyselective channel, ICI, as well as ISI, can effectively be avoided by inserting a cyclic prefix before each block of parallel data symbols at the cost of power loss and bandwidth expansion [5, 6].

Many researches were proposed to improve the performance of the OFDM system such as channel equalization [7-9] and ICI reduction using pre-coding for self-cancelation[10-14] and using wideband OFDM with spectrum losses[15,16]. In [1719] a DHT based OFDM system is proposed similarly as the conventional system except the FFT is replaced by the DHT to improve system performance. In this paper a suitable solution is proposed to improve system performance through the splitting of OFDM spectrum into multi-orthogonal bands where each band is designed to carry local OFDM symbol and all local symbols form the global OFDM symbol.

\section{DISCRETE HARTLEY TRANSGORM}

The Hartley transform is very simple transform that significantly related to the Fourier transform, but with real valued mapping, where real values are transformed simply to real values in the Hartley domain. It was proposed by Ralph Vinton Lyon Hartley in 1942, instead of the Fourier transform and is one of the famous Fourier-related transforms.

The Hartley transform of a function $\mathrm{f}(\mathrm{t})$ can be expressed as $\mathrm{H}(\mathrm{w})=\frac{1}{\sqrt{2 \pi}} \int_{-\infty}^{\infty} \mathrm{f}(\mathrm{t}) \operatorname{Cas}(\mathrm{wt}) \mathrm{dt} \quad(1)$

where the Cas function, is defined as:

$\operatorname{Cas}(w t)=\operatorname{Cos}(w t)+\operatorname{Sin}(w t)$

The inverse Hartley transform can be defined as

$f(t)=\frac{1}{\sqrt{2 \pi}} \int_{-\infty}^{\infty} H(w) \operatorname{Cas}(w t) d w(3)$

From the forward and backward transformation equations (eq.1 and eq.3), one can see that, the inverse transformation (synthesis equation) has the same mathematical operations as the forward transformation (analysis equation). The coefficient $1 / \sqrt{2 \pi}$, selected by Hartley for the forward and inverse transformations, is used to achieve self-inverse condition [20, 21].

The Hartley transform has the advantages of real to real functions transformation (as opposed to requiring complex numbers in the case of Fourier transform) and the self-inverse property as compared with the Fourier transform. The Discrete Hartley Transform (DHT) was proposed in the early 1980s by Wang and Bracewell. Before this time The Hartley transform received little interest [22, 23]. The DHT can becalculated efficiently with transform matrix operations as in the DFT. This leads to FFT related fast algorithms, where the Fast Hartley Transform (FHT) can be computed from the FFT, and vice versa [22]. 
The real function ( cas ) in the Hartley transform and the complex exponential term in the Fourier transform is the main difference between the two transforms. As well known, the real computations are much simpler than complex mathematical operations; therefore the FHT is faster than the FFT and requires fewer floating-point operations and hence the run times and memory requirements will be less for signal processing with FHT as compared with FFT processing. Moreover, a given signal is transformed using FHT with fewer operations, so there are fewer round-off errors [24].

The forward and inverse discrete Hartley transform pair is given by [23]:

$X_{H}(k)=\sum_{n=0}^{N-1} x(n) \operatorname{cas}\left(\frac{2 \pi n k}{N}\right)$

$k=0,1,2, \ldots \ldots, N-1$

$x(n)=\frac{1}{N} \sum_{k=0}^{N-1} X_{H}(k) \operatorname{cas}\left(\frac{2 \pi n k}{N}\right)$

$n=0,1,2, \ldots \ldots, N-1$

Where

$\operatorname{cas} \varphi=\cos \varphi+\sin \varphi$

The sequence $X_{H}(k)$ is periodic with period $N$ similarly as with the DFT.

From the above equations one can note the self-inverse operation, where the same computer program or system can be used to perform the forward and inverse transformation. This is not the case for the DFT, where a complex output may be obtained from real-valued signal transformation.

Many researches were proposed to compute the Discrete Hartley Transform using Fast Hartley Transform algorithm (FHT) [25 - 27]. The discrete Hartley transform for a length $\mathrm{N}$ sequence $h(n)=\left[h_{0} h_{1} \ldots \ldots . . h_{N-I}\right]$ can be computed in matrix form as [27]:

$\left[\begin{array}{c}H_{0} \\ H_{1} \\ H_{2} \\ \vdots \\ H_{N-1}\end{array}\right]=\left[\begin{array}{ccccc}1 & 1 & 1 & \cdots & 1 \\ 1 & \operatorname{cas}(1) & \operatorname{cas}(2) & \cdots & \operatorname{cas}(N-1) \\ 1 & \operatorname{cas}(2) & \operatorname{cas}(4) & \cdots & \operatorname{cas}(2(N-1)) \\ \vdots & \vdots & \vdots & \ddots & \vdots \\ 1 & \operatorname{cas}(N-1) \operatorname{cas}(2(N-2)) \cdots \operatorname{cas}((N-1)(N-1))\end{array}\right]\left[\begin{array}{c}h_{0} \\ h_{1} \\ h_{2} \\ \vdots \\ h_{N-1}\end{array}\right](7)$

for the sake of simplicity, they denote $\operatorname{cas}\left(\frac{2 \pi}{N} k n\right)$ by $\operatorname{cas}(k n)$ i.e

$\mathrm{H}(\mathrm{k})=[\mathrm{T}] \times \mathrm{h}(\mathrm{n})(8)$

Where [T]: is the Discrete Hartley Transform matrix.

As we know that DHT has identical inverse so IDHT and DHT matrices are same, (i.e. $[\mathrm{T}]=[\mathrm{T}]^{-1}$ ) so

$\mathrm{h}(\mathrm{n})=[\mathrm{T}]^{-1} \times \mathrm{H}(\mathrm{k})=[\mathrm{T}] \times \mathrm{H}(\mathrm{k})(9)$
$[\mathrm{T}]=\left[\begin{array}{ccccc}1 & 1 & 1 & \cdots & 1 \\ 1 & \operatorname{cas}(1) & \operatorname{cas}(2) & \cdots & \operatorname{cas}(N-1) \\ 1 & \operatorname{cas}(2) & \operatorname{cas}(4) & \cdots & \operatorname{cas}(2(N-1)) \\ \vdots & \vdots & \vdots & \ddots & \vdots \\ 1 & \operatorname{cas}(N-1) \operatorname{cas}(2(N-2)) & \cdots & \operatorname{cas}((N-1)(N-1))\end{array}\right](10)$

\section{PROPOSEDHARTLEYBASEDMULTI- ORTHOGONALBAND OFDM SYSTEM}

The conventional OFDM system is shown in Figure 1. Each sub-carrier is modulated in amplitude and phase by the data bits. Modulation techniques typically used are Quadrature Phase Shift Keying (QPSK), Quadrature Amplitude Modulation (QAM), 16-QAM, 64-QAM etc., The process of combining different subcarriers to form a composite timedomain signal is achieved using Fast Fourier Transform (FFT) and Inverse FFT (IFFT) operations. Thus, the FFT-based OFDM use the IFFT algorithm to generate the required orthogonality between subcarriers.

As well known, Multi-path fading channels have a severe effect on the orthogonality between subcarriers and hence system performance degrades. In this paper a suitable solution is proposed to improve the system performance through the splitting of OFDM spectrum into multi-orthogonal local bands. In each local band the DHT is used to produce the orthogonality between the sub-carriers and can be considered as a local OFDM symbol. Finally the global OFDM symbol is formed from all local spectrums together. Also, the IDHT is used to produce the orthogonality between carriers in the global OFDM symbol. The proposed system is shown below in Figure 2 where the transmitter is shown in (a) and the receiver is shown in (b).

Let us consider the total number of carriers and data symbols equal to $(N)$. Consider the data stream $d=\left[\begin{array}{llll}d_{1} & \ldots \ldots . . d_{N}\end{array}\right]^{T}$ is divided into $(m)$ groups $D g_{l}(n), D g_{2}(n) \ldots \ldots \ldots, D g_{m}(n)$, where each group has $N / m$ carriers

$D g_{1}(n)=\left[\begin{array}{lll}d_{1} & \left.\ldots . . d_{N / m}\right]^{T}\end{array} ; \quad D g_{2}(n)=\left[d_{(N / m)+1} \ldots \ldots . . d_{2 N / m}\right]^{T} \quad ;\right.$ $\ldots \ldots \ldots \ldots ; D g_{m}=\left[d_{N-(N / m)+1} \ldots \ldots . . d_{N}\right]^{T}$;

The data of each group considered a time domain symbols converted to frequency domain using the discrete Hartley transform

$D g_{H}(k)=\sum_{n=0}^{N / m-1} D g(n) \operatorname{cas}\left(\frac{2 \pi n k}{(N / m)}\right)$

$k=0,1,2, \ldots \ldots,(N / m)-1$

Or in matrix form as given in eq. (8):

$D g(k)=[T] \times D g(n) \quad(12)$

Where $[T]$ is $(\mathrm{N} / \mathrm{m})$ by $(\mathrm{N} / \mathrm{m})$ Hartley matrix obtained from eq. (10) by replacing each $(N)$ by $(N / m)$. In the next step; all groups data combined together to form a frequency domain vector of length $\mathrm{N}$;

$D(k)=\left[D g_{1 H}(k) D g_{2 H}(k) D g_{3 H}(k) \quad D g_{m H}(k)\right]^{T}$

where each $D_{g H}(k)$ is a vector of length $(\mathrm{N} / \mathrm{m})$

where 


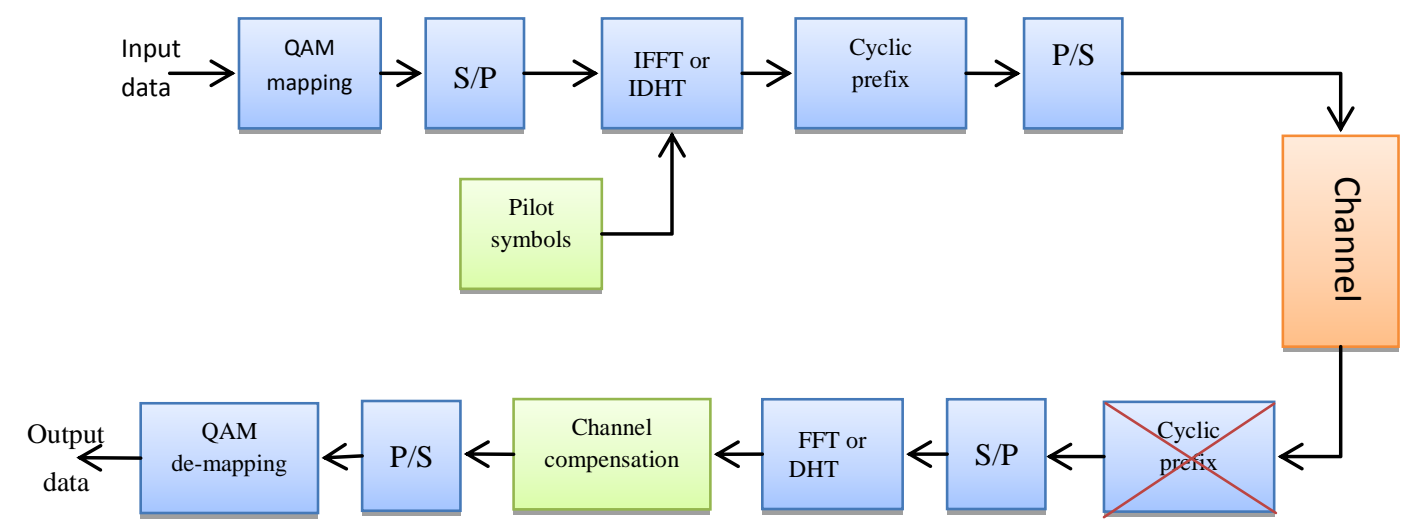

Fig 1: The block diagram of the conventional OFDM System

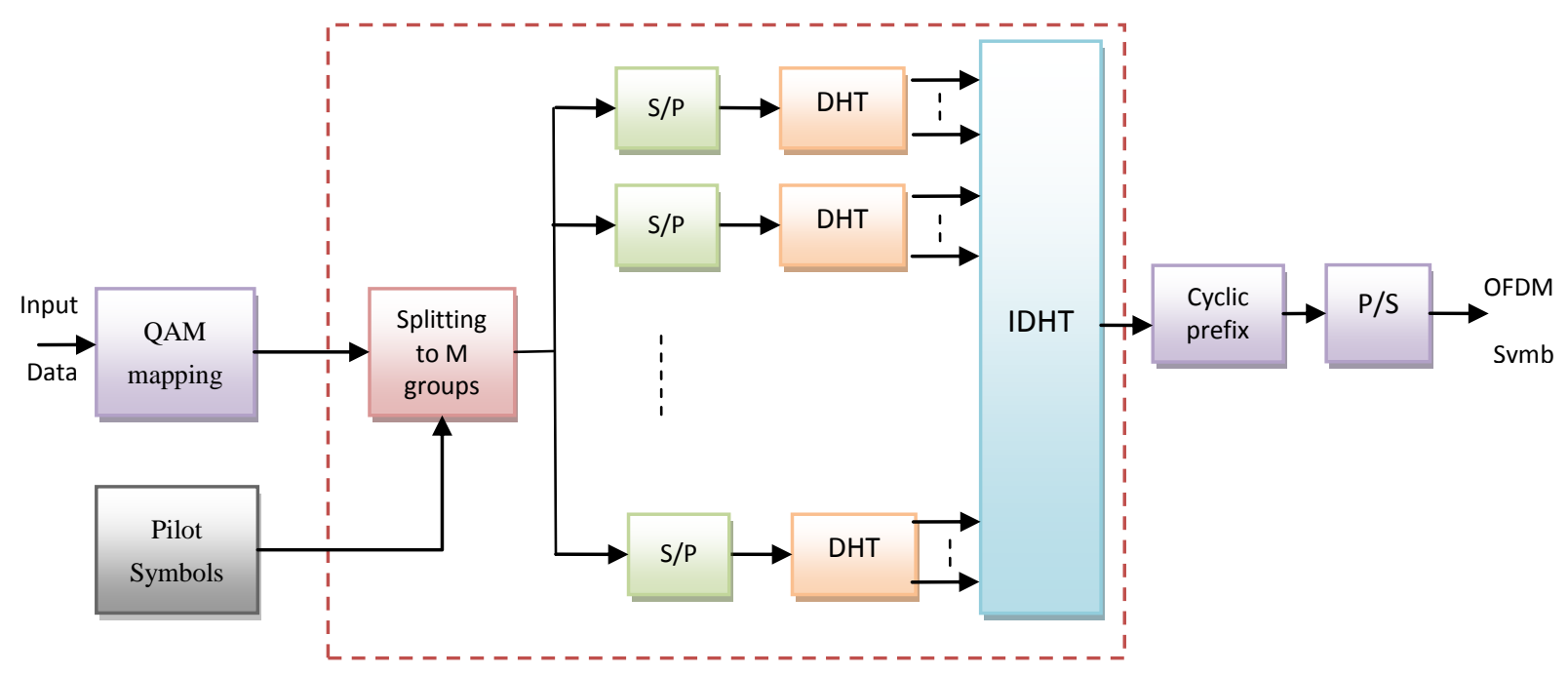

(a)

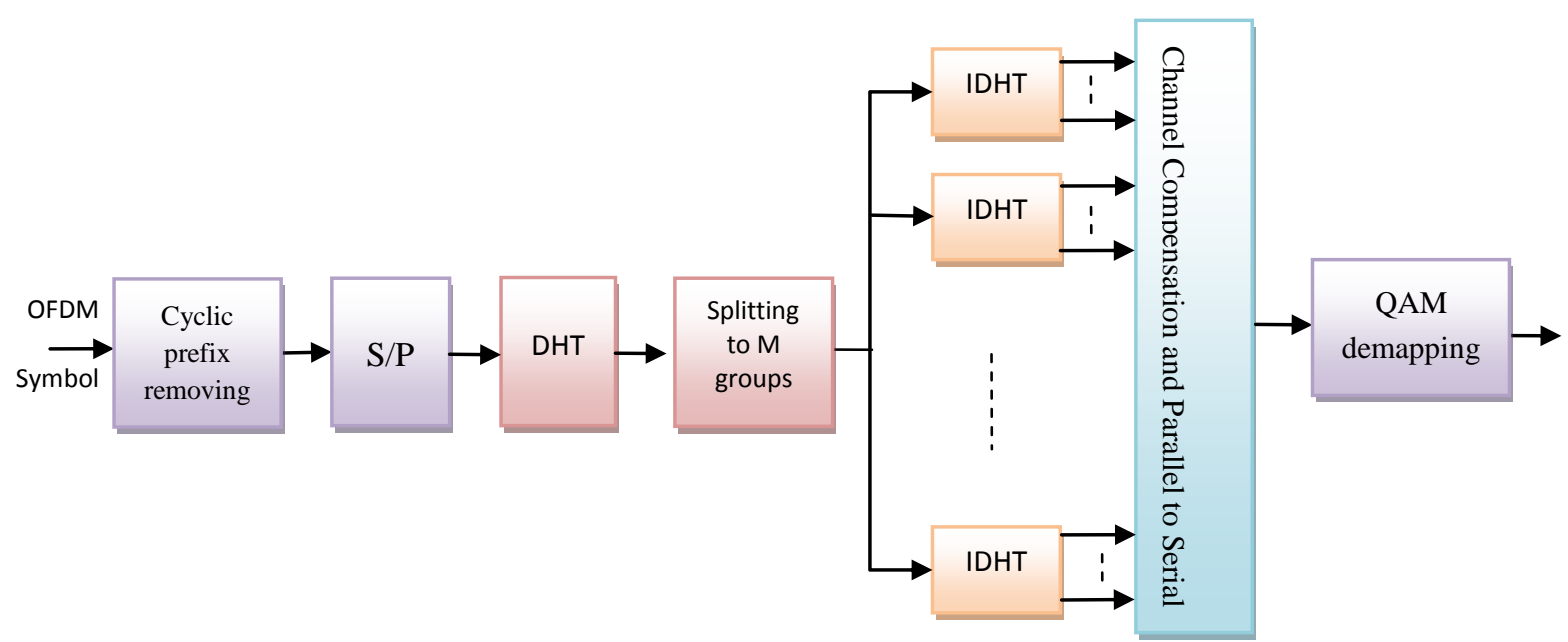

(b)

Figure 2: The proposed Multi Orthogonal Band-OFDM a: transmitter b: receiver 
Finally; the OFDM symbol is formed by taking the inverse discrete Hartley transform to the vector $\mathrm{D}(\mathrm{k})$

$$
\begin{aligned}
& Y_{\text {OFDM }}(n)=\frac{1}{N} \sum_{k=0}^{N-1} D(k) \operatorname{cas}\left(\frac{2 \pi n k}{N}\right) \\
& n=0,1,2, \ldots \ldots, N-1
\end{aligned}
$$

The receiver is designed to perform the inverse process of the transmitter as shown in Figure 2 (b). The suitability of Hartley transform in this system stems from the fact that the Hartley transform has real computations;and real arithmetic is much simplerthan complex computation; the DHT is faster than the DFT and requires fewer floating-point operations, which implies faster run-times and less computer memory to process a signal in comparison to a typical FFT algorithm. The FHT uses fewer operations to transform a given signal, so there are fewer round-off errors.

The DHT is self-inverse, which means that the same computer program or hardware can be used for the forward and inverse transform. This is not the case for the DFT, where a realvalued signal is transformed into a complex spectrum.

\section{SIMULATION AND PERFORMANCE ANALYSIS OF THE HARTLEY BASED MULTI-ORTHOGONAL-BAND OFDM}

In this section the conventional and the proposed OFDM systems are simulated using MATLAB and BER performance evaluated for different channel conditions. The system parameters that are used through simulation are: bit rate equal $4 * 10^{6} \mathrm{bit} / \mathrm{sec}$; the mapper is $16-\mathrm{QAM}$ mapper to make the symbol rate $=10^{6}$ symbol/sec.; FFT bins (for conventional $\mathrm{OFDM})=64$. For the proposed model DHT bins $=64$ and divided intoeight groups each group contain $(8$ bins) i.e. $\{\mathrm{N}=64, \mathrm{~m}=8, \mathrm{~N} / \mathrm{m}=8 \mathrm{MOB}-\mathrm{OFDM}\}$.

The cyclic prefix equal to $0.25 *$ FFT bins $=0.25 *$ DHT bins $=16$. Different types of channel models are taken into account during simulation. First, an AWGN channel is considered with several SNR values. Then,multi-path Rayleigh distributed flat and selective fading channels are considered.

Figure 3 illustrates the performance of the conventional FFT based and MOB-Hartley based OFDM systems in AWGN channel. From Figure 3 it can be seen that the FFT-based OFDM need higher SNR than the proposed MOB-OFDM to achieve the same BER performance, where the proposed system gives a gain in SNR about $(9.5 \mathrm{~dB})$ to achieve a BER performance of $10^{-4}$ as compared with the conventional OFDM system.

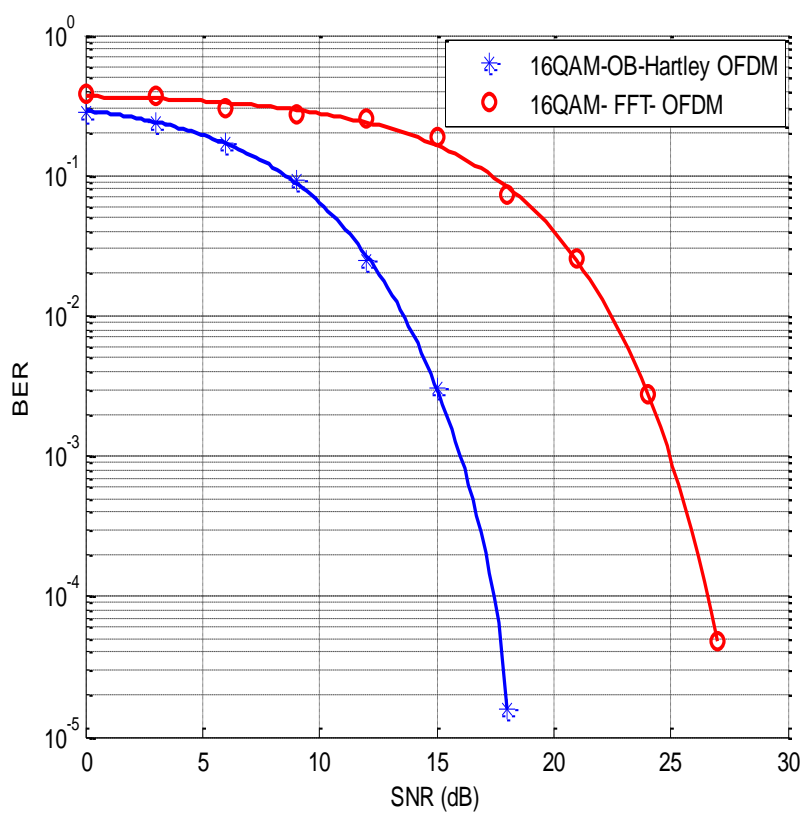

Fig 3: BER Performance in AWGN channel

Secondly; a flat fading channel addition to AWGN is considered; in this case all the frequency components in the signal are affected by a constant attenuation and linear phase distortion of the channel, which has been chosen to have a Rayleigh's distribution. A Doppler frequency of $50 \mathrm{~Hz}$ is used in this simulation. Figure (4) shows the BER performance of the proposed MOB-Hartley based OFDM and standard FFT based OFDM in this channel. From this figure, it can be seen that the proposed OFDM has better performance than the standard OFDM. This is due to the good orthogonallity obtained in the proposed method. It can be seen that MOBHartley OFDM gives a gain in SNR of about $9 \mathrm{~dB}$ to achieve a BER performance of $10^{-3}$ as compared with standard FFT based OFDM.

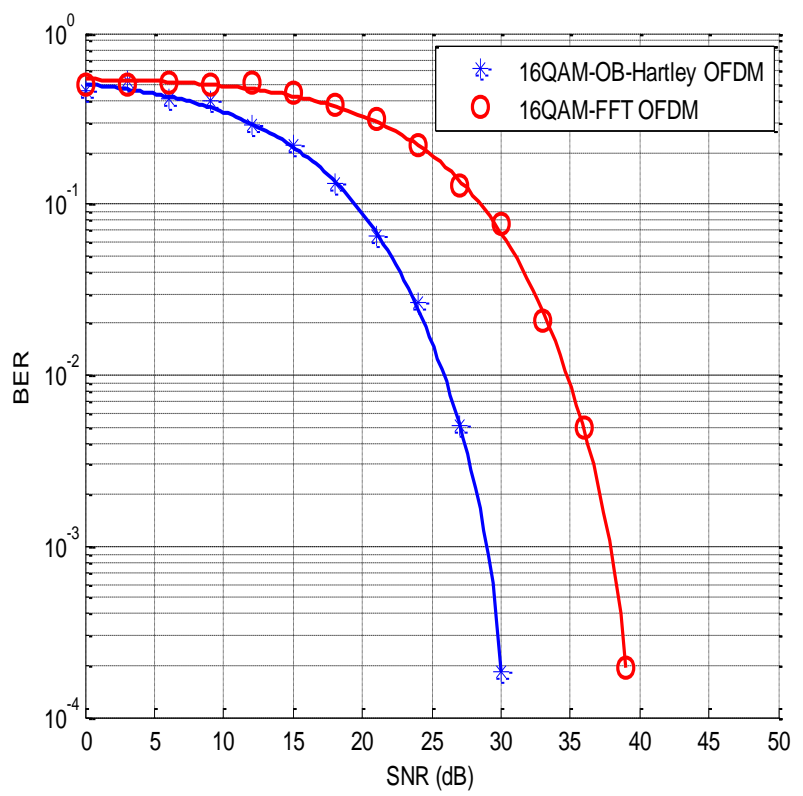

Fig 4: BER Performance in flat fading channel 
Figure (5) show the BER performance of the MOB-Hartleybased OFDM and standard FFT OFDM in selective fading channel. A 2-ray Rayleigh-distributed multi-path fading channel with Doppler frequency of $50 \mathrm{~Hz}$ is assumed, the attenuation and delay of the second path are $-8 \mathrm{~dB}$ and 3 samples respectively. From Figure (5) it can be seen that, the performance of MOB-Hartley based OFDM still better than the performance of standard OFDM where the proposed MOB-OFDM gives a gain in SNR about $6 \mathrm{~dB}$ to achieve a BER performance of $10^{-3}$ as compared with standard FFT based OFDM.

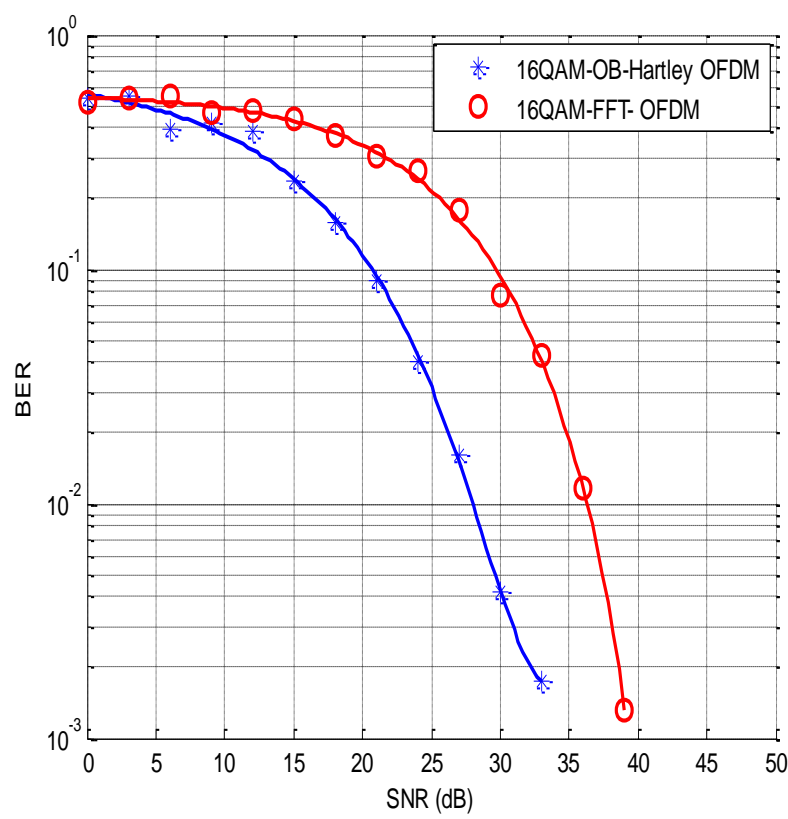

Fig 5: BER Performance in selective fading channel

The effect of variation of selective fading channel parameters on the performance of the proposed system is studied. The first test gives the effect of changing the $2^{\text {nd }}$ path gain on the $\mathrm{BER}$, and the second test gives the effect of changing $2^{\text {nd }}$ path signal delay on the BER performance.

Figure (6) illustrates the BER performance of OB-Hartley and FFT based systems with 16 QAM and 64QAM mappers as a function for attenuation of the second path, the attenuation varies from 0.1 to 1 (from $-10 \mathrm{~dB}$ to $0 \mathrm{~dB}$ ). During this simulation the SNR, $2^{\text {nd }}$ path delay and the Doppler frequency kept constant at $(35 \mathrm{~dB}, 3$ samples, $50 \mathrm{~Hz})$ respectively.

From the observation of the results obtained in Figure6, it's clearly seen the effect of the 2nd path gain on the BER performance that is the BER becomes worse when the path gain is increased, any way the BER performance of the proposed OFDM system still better than the standard OFDM system.

Figure (7) illustrates the BER performance as a function of delay of the second path, the delay varies from 1 to 15 in samples. During this simulation the SNR, $2^{\text {nd }}$ path attenuation and the Doppler frequency are kept constant at $(35 \mathrm{~dB},-8 \mathrm{~dB}$, $50 \mathrm{~Hz}$ ) respectively. From Figure (7), the BER will increase with increasing the delay, but the increase in the proposed OFDM will be less than that for FFT based-OFDM.

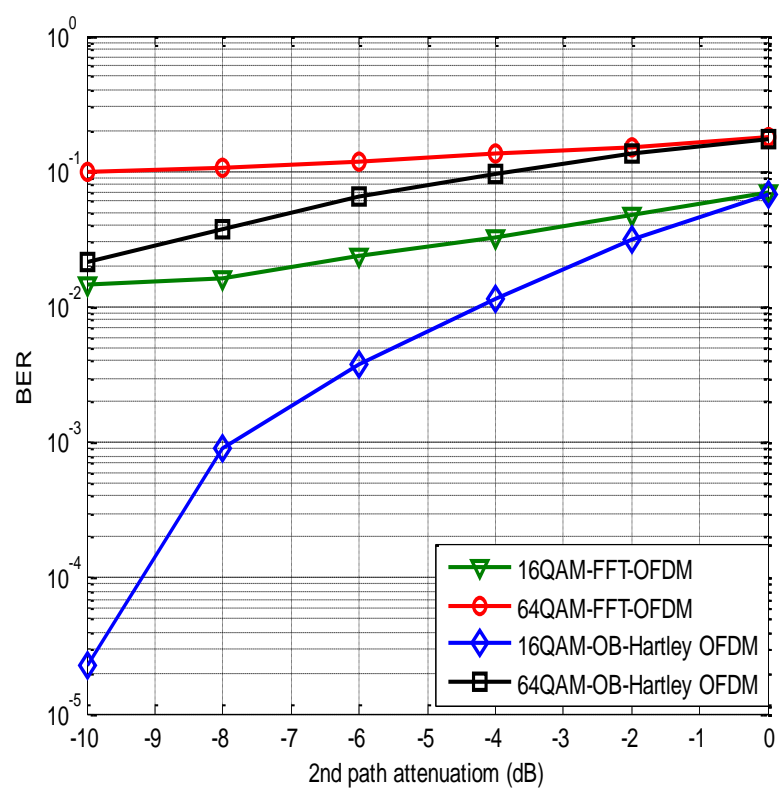

Fig 6: BER versus attenuation of the second path

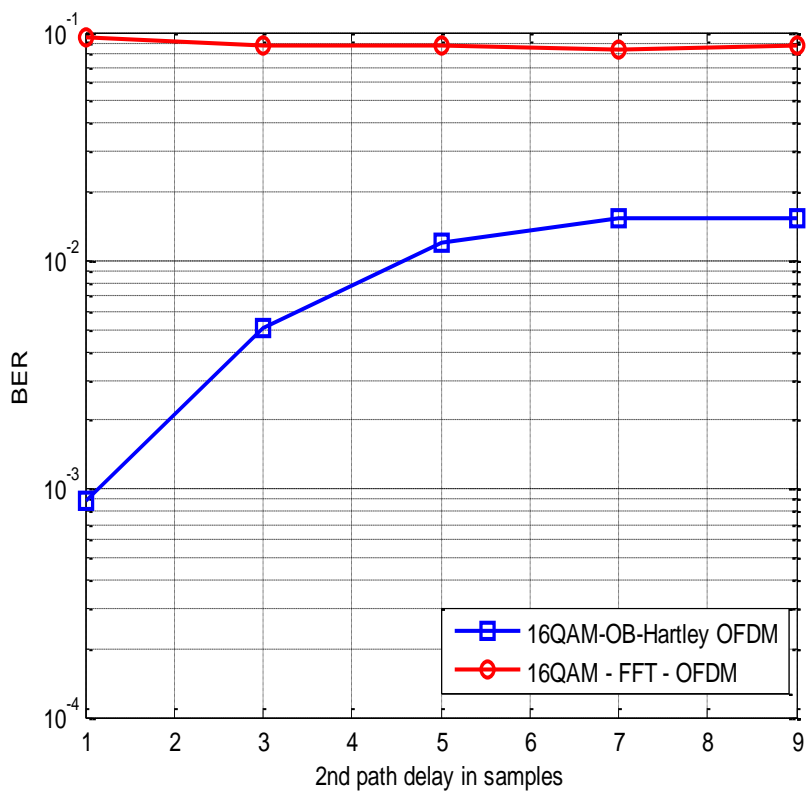

Fig 7: BER versus delay of the second path

The Peak to Average Power Ratio (PAPR) for both MOBHartley and FFT based-OFDM systems is simulated and compared. The PAPR is a random variable for each OFDM symbol. Table 1 show that the PAPR for the two systems. The results correspond to the average of the PAPR for many OFDM symbols. From this table, one can see that the PAPR of the proposed OFDM is approximately the same as of the standard FFT-OFDM

The time domain signal envelope of the proposed and conventional systems shown in figure (8) gives the main reason for the similarity in the PAPR results. 
Table 1: Peak to Average Power Ratio (PAPR) results

\begin{tabular}{|l|c|}
\hline \multicolumn{1}{|c|}{ System } & PAPR \\
\hline 16-QAM OB-Hartley based OFDM & $11.6229 \mathrm{~dB}$ \\
\hline 16-QAM FFT based OFDM & $11.8026 \mathrm{~dB}$ \\
\hline 64-QAM OB-Hartley based OFDM & $11.8041 \mathrm{~dB}$ \\
\hline 64-QAM FFT based OFDM & $12.0762 \mathrm{~dB}$ \\
\hline
\end{tabular}

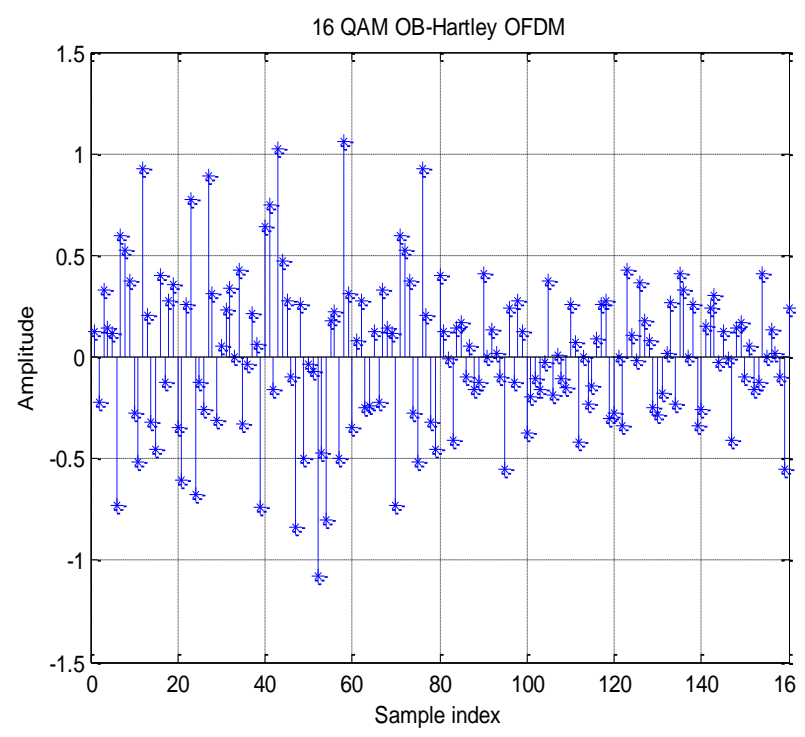

(a)

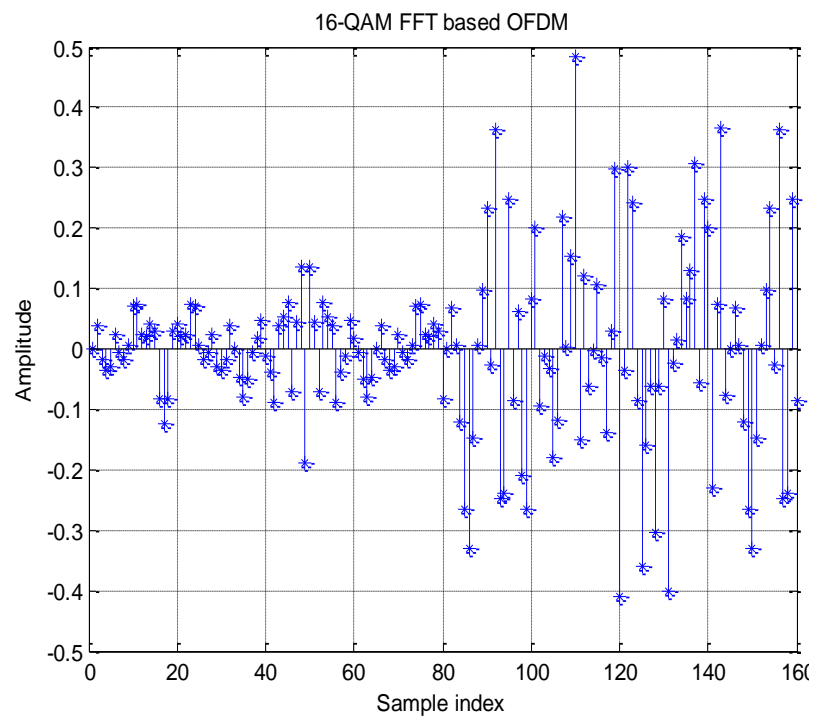

(b)

Fig 8: OFDM signal envelope: a- 16 QAM OB-Hartley OFDM

b- 16 QAM FFT based OFDM

\section{CONCLUSIONS}

In this papera BER performance improvement is proposed for OFDM system based on the splitting of OFDM spectrum into multi-orthogonal local bands to form a local OFDM symbols all used to generate the global OFDM symbol.The DHT is used to generate the subcarriers twice one in the local symbols and secondly in the global symbol. The appropriateness of the Hartley transform in the proposed system came from its characteristics, where the DHT is self-inverse transform and has real computationswith fewer round-off errors so that the transformation can be faster and simpler as compared with the complex computations of the FFT. The proposed MOBOFDM system is simulated and compared with the conventional FFT-OFDM system under different channel conditions. As a results the proposed system improves the BER performance and keeps the bandwidth and PAPR same as in the conventional FFT-OFDM system. The performance improvement achieved through the orthogonality increasing due to the twice DHT using in the transmitter.

Approximately, a SNR gain of $9 \mathrm{~dB}$ is gained to achieve an error of $10^{-3}$ in AWGN and flat fading channels. While in multi-path frequency selective channel a SNR gain of $6 \mathrm{~dB}$ is gained to achieve such an error assuming 2-ray Rayleighdistributed multi-path fading channel with $2^{\text {nd }}$ path delay of 3 samples and attenuation of $-8 \mathrm{~dB}$.

\section{REFERENCES}

[1] Veena M. B. \& Dr. M.N.Shanmukha S. 2011," Performance Analysis of DWT Based OFDM Over FFT Based OFDM and Implementing on FPGA", International Journal of VLSI design \& Communication Systems (VLSICS) Vol.2, No.3.

[2] Aida Zaier and RidhaBouallègue 2011, "A Full Performance Analysis of Channel Estimation Methods for Time Varing OFDM Systems", International Journal of Mobile Network Communications \& Telematics (IJMNCT) Vol.1, No.2.

[3] SangeetaJajoria, Sajjan Singh and S. V. A. V. Prasad 2012," Analysis of BER Performance of OFDM System by Adaptive Modulation", International Journal of Recent Technology and Engineering (IJRTE) ISSN: 2277-3878, Volume-1, Issue-4

[4] Cimini L. 1985, "Analysis and Simulation of a Digital Mobile Channel Using Orthogonal Frequency Division Multiplexing," Computer Journal of IEEE Transactions on Communication, vol. 33, no. 3, pp. 665-675

[5] Weinstein S. and Ebert P. 1971, "Data Transmission by Frequency Division Multiplexing Using the Discrete Fourier Transform," Computer Journal of IEEE Transactions Communications Tech, vol. 19, no. 3, pp. 628-634.

[6] Abbas Kattoush 2010," A Novel Radon-Wavelet Based OFDM System Design and Performance Under Different Channel Conditions", The International Arab Journal of Information Technology, Vol. 7, No. 4.

[7] SinemColeri, Mustafa Ergen, AnujPuri, and Ahmad Bahai 2002, "Channel Estimation Techniques Based on Pilot Arrangement in OFDM Systems", IEEE Transactions on Broadcasting, VOL. 48, NO. 3. 
[8] Jan-Jaap van de Beek, OveEdfors, Magnus Sandell,Sarah Kate Wilson and Per Ola B.rjesson 1995, " On Channel Estimation In OFDM Systems", In Proceedings of Vehicular Technology Conference (VTC 95), vol. 2, pp. 815-819, Chicago, USA, September 1995

[9] H'ectorPoveda, Guillaume Ferr'e and Eric Grivel 2012, "Frequency Synchronization and Cannel Equalization for an OFDM-IDMA Uplink System", Icassp 2012, Kyoto : Japan.

[10] Yi-HaoPeng, Ying-ChihKuo, Gwo-Ruey Lee and Jyh-Horng Wen 2007," Performance Analysis of a New ICI-Self-Cancellation-Scheme in OFDM Systems", IEEE Transactions on Consumer Electronics, Vol. 53, No. 4.

[11] MiftahurRahman, PreyomKantiDey, and M. F. Rabbi Ur Rashid 2012," Improved ICI Self Cancellation Scheme for Phase Rotation Error Reduction in OFDM System", International Journal of Information and Electronics Engineering, Vol. 2, No. 4.

[12] Yang Cao, Yong Ren and Xiuming Shan 2003," Frequency Offset Correction for PCC-OFDM with Overlapping Symbol Periods" Proceedings of ICCT2003

[13] Yuping Zhao and Sven-Gustav Häggman 2001, "Intercarrier Interference Self-Cancellation Scheme for OFDM Mobile Communication Systems", IEEE Transactions on Communications, VOL. 49, NO. 7.

[14] Y.H. Peng et al. 2007, "Performance Analysis of a New ICI-Self-Cancellation-Scheme in OFDM Systems", IEEE Transactions on Consumer Electronics, Vol. 53, No. 4.

[15] A. Batra, J. Balakrishnan, G. R. Aiello, J. R. Foerster and A. Dabak 2004, "Design of a Multiband OFDM System for Realistic UWB Channel Environments," IEEE Transactions on Microwave Theory and Techniques, Vol. 52, Issue 9, pp. 2123-2138.

[16] Hao $\mathrm{Lu}$ 2013, "Cooperative Wideban OFDM Communication". Thesis -Delft University of Technology.
[17] Chin-KuoJao, Syu-Siang Long, and MuhTianShiue 2010, "DHT-Based OFDM System for Passband Transmission Over Frequency-Selective Channel", IEEE Signal Processing Letters, Vol. 17, NO. 8, pp.699-702.

[18] ZakariaSembiring, MohdFareqAbdMalek, and Hasliza Rahim 2011, “A Low Complexity OFDM Modulator and Demodulator Based On Discrete Hartley Transform", 2011 Fifth Asia Modelling Symposium, pp.252-256, DOI 10.1109/AMS.2011.

[19] Vijay Kumar, Ganapati Panda, and Prasant Kumar Sahu 2009, "DHT Based 4 QAM OFDM Baseband System and Channel Estimation", International Journal of Recent Trends in Engineering, Vol 2, No. 5, pp.80-48.

[20] Ed. Alexander \& D. Poularikas 2000, "The Transforms and Applications Handbook", $2^{\text {nd }}$ Edition, Boca Raton: CRC Press LLC.

[21] R. N. Bracewell 1986, "The Hartley Transform", New York, Oxford Univ. Press.

[22] Alfred Mertins 1990, "Signal Analysk: Wavelets, Filter Banks, Time-Frequency Transforms and Applications" John Wiley \& Sons Ltd.

[23] R. N. Bracewell 1983, "Discrete Hartley transform," J. Opt. Soc. Am. 73 (12), PP. 18321835 .

[24] Mark Hyosun Mar 1990 "The Fast Hartley Transform", Harry Diamond Laboratories HDLTM-90-23.

[25] Bracewell, R.N 1984, “The Fast Hartley Transform", Proceedings of the IEEE, vol. 72, No. 8, pp. 1010-1018.

[26] de Oliveira, H.M., Campello de Souza, R. M. 2001, "A Fast Algorithm for Computing the Hartley/Fourier Spectrum", Proceeedings of the Brazilian Academy of Science, Rio de Janeiro, vol. 73, pp.468-468.

[27] Sorensen, S. V., Jones, D. L., Burrus, C. S. and Heideman, M. T. 1985, "On Computing the Discrete Hartley Transform”, IEEE Trans. Acoust., Speech and Signal Processing, vol. ASSP 33, No. 4, pp. 1231-1238. 